\title{
Atmospheric polarimetric effects on GNSS radio occultations: the ROHP-PAZ field campaign
}

\author{
R. Padullés ${ }^{1}$, E. Cardellach ${ }^{1}$, M. de la Torre Juárez ${ }^{2}$, S. Tomás ${ }^{1}$, F. J. Turk ${ }^{2}$, S. Oliveras ${ }^{1}$, C. O. Ao ${ }^{2}$, and A. Rius ${ }^{1}$ \\ ${ }^{1}$ Institut de Ciències de l'Espai (ICE - CSIC / IEEC), Barcelona, Spain \\ ${ }^{2}$ NASA Jet Propulsion Laboratory, California Institute of Technology, Pasadena, CA, USA \\ Correspondence to: R. Padullés (padulles@ice.cat)
}

Received: 14 May 2015 - Published in Atmos. Chem. Phys. Discuss.: 9 July 2015

Revised: 19 November 2015 - Accepted: 15 December 2015 - Published: 21 January 2016

\begin{abstract}
This study describes the first experimental observations showing that hydrometeors induce polarimetric signatures in global navigation satellite system (GNSS) signals. This evidence is relevant to the PAZ low Earth orbiter, which will test the concept and applications of polarimetric GNSS radio occultation (RO) (i.e. ROs obtained with a dualpolarization antenna). A ground field campaign was carried out in preparation for PAZ to verify the theoretical sensitivity studies on this concept (Cardellach et al., 2015). The main aim of the campaign is to identify and understand the factors that might affect the polarimetric GNSS observables. Studied for the first time, GNSS signals measured with two polarimetric antennas ( $H$, horizontal, and $V$, vertical) are shown to discriminate between heavy rain events by comparing the measured phase difference between the $H$ and $V$ phase delays $(\Delta \Phi)$ in different weather scenarios. The measured phase difference indicates higher dispersion under rain conditions. When individual events are examined, significant increases in $\Delta \Phi$ occur when the radio signals cross rain cells. Moreover, the amplitude of such a signal is much higher than the theoretical prediction for precipitation; thus, other sources of polarimetric signatures have been explored and identified. Modelling of other hydrometeors, such as melting particles and ice crystals, have been proposed to explain the obtained measurements, with good agreement in more than $90 \%$ of the cases.
\end{abstract}

\section{Introduction}

Global navigation satellite system (GNSS) radio occultation (RO) spaceborne missions have been probing the Earth's atmosphere since 1995 (e.g. Rocken et al., 1997). They have been shown to be useful for climate monitoring (e.g. Steiner et al., 2011), and nowadays their thermodynamic profiles are being assimilated operationally into several numerical weather prediction (NWP) models (e.g. Healy et al., 2005; Cucurull and Derber, 2008).

A new measurement concept presented in Cardellach et al. (2015) aims at detecting and quantifying heavy precipitation events using polarimetric GNSS RO by means of measuring the difference between the phase delays of the horizontal and the vertical components of the received propagated signal. This technique will be tested aboard the PAZ low Earth orbiter (LEO) satellite during the RO and heavy precipitation experiment (ROHP-PAZ), and it will be the first attempt to detect rain using L-band frequencies $(1.575 \mathrm{GHz}$, i.e. $\lambda=19.03 \mathrm{~cm}$ ). The launch is planned for 2016 . The theoretical analysis performed in Cardellach et al. (2015) demonstrated not only that heavy rain events could be detected but also that an approximated vertical structure of the rain cells could be retrieved.

Prior to the launch of the PAZ satellite, a field campaign was conducted in order to study, for the first time, GPS signals obtained at two polarizations in grazing angle geometry. The goal was to start identifying and understanding the factors that might affect the polarimetric signal. Positioned at the top of a mountain at $1670 \mathrm{~m}$ a.m.s.l., the experiment set up consisted of the engineering model of the PAZ's polarimetric antenna pointing at the horizon and a commercial Javad receiver (provided by the German Research Center for Geosciences, GFZ, and enclosed in a shelter). A zenithlooking geodetic GNSS antenna was also used for positioning. The RO antenna pointed south and to the horizon, and it tracked all the visible satellites in the east-west field of view from -5 to $40^{\circ}$ of elevation and from 150 to $270^{\circ}$ of 
azimuth (see Fig. 1). Although all the satellites were tracked simultaneously, only those crossing the main beam of the antenna were used in the posterior analysis. For the time period analysed, the GNSS satellites with the highest number of samples are the ones identified by the pseudo-random noise (PRN) numbers G10, G14, G15, G22 and G31. Moreover, only the segments between 0 and $20^{\circ}$ of elevation are used for the analysis, since the antenna performance reaches its optimal values within this range. Given the geometry of the experiment's field of view, in most of the cases only one of either the descending or ascending trajectories over the horizon provided data within the antenna field of view.

The main objective was to collect a large amount of data free of rain and to catch some heavy rain events in order to observe differences in the polarimetric observables between the two data sets. The area was chosen specifically for this purpose, given that the region is mainly dry and several intense local Mediterranean storms occur a few times per year (Ducrocq et al., 2014). The experiment ran for 7 months, from 21 March to 10 October in 2014. During this period, it collected data for about 170 days. There were about 25 days of rain, of which 5 could be considered heavy rain.

The geometry and measurements used for this experiment are closely related to those of polarimetric weather radar observations. In the radar observations, the differential reflectivity $\left(Z_{\mathrm{dr}}\right)$ and the specific differential phase $\left(K_{\mathrm{dp}}\right)$ are the most important polarization signatures for rain characterization at low elevation angles (e.g. Bringi and Chandrasekar, 2001). Some differences apply in this case: we are limited to one single observable, the differential phase between the horizontal $(H)$ - and vertical $(V)$-port phase delay $(\Delta \Phi)$, defined as

$\Delta \Phi=\int_{L} K_{\mathrm{dp}} \mathrm{d} l$,

where $L$ is the path length under the influence of $K_{\mathrm{dp}}$. The $K_{\mathrm{dp}}$ can be understood as a measure of the bi-refraction induced by hydrometeors that have an asymmetry between the horizontal and vertical axis. Note also that $K_{\mathrm{dp}}$ is here defined in propagation (forward-scattering) rather than backscattering. This is a one-dimensional observation, since it is an integral along the ray path. Furthermore, the weather radars work with frequencies equal to or higher than $3 \mathrm{GHz}$; thus, the sensitivity to hydrometeors is expected to be higher than for the L-band signals.

From the polarimetric radar observations it is known that different kinds of rain, precipitation and particles could produce different $K_{\mathrm{dp}}$. Studies for rain (e.g. Bringi and Chandrasekar, 2001; Trömel et al., 2013), ice (e.g. Vivekanandan et al., 1994; Ryzhkov and Zrnić, 1998), snow (e.g. Matrosov, 1992; Kennedy and Rutledge, 2011) and melting layer (e.g. Baldini and Gorgucci, 2006; Trömel et al., 2013) characterization using polarimetric observables have been conducted extensively, as has the continuous satellite observation of rain such as the Tropical Rainfall Measurement Mission (TRMM) and the Global Precipitation Measurement (GPM) mission. Therefore, the aim here is not to characterize the different kinds of precipitation or hydrometeors but to take advantage of this knowledge to understand the observations.

This paper is organized in the following way: in Sect. 2 the experiment geometry and the acquired data are described in detail and a comparison with the satellite set-up is performed. Problems with the signal, local multipath characterization, and expected improvements from satellite observations are addressed here. The collocated meteorological data used for validation are described in Sect. 3. The statistical results of the experiment are shown in Sect. 4, and a comparison with the forward model simulation results is performed in Sect. 5 . Finally, in Sect. 6 the conclusions are discussed.

\section{Polarimetric GNSS data}

\subsection{Observables}

GNSS signal observables are the carrier phase and the pseudo-range. In the standard RO, these are measured with a circular co-polar antenna (right-handed, as in the transmitted signals), and they are used to obtain the bending angle, which in turn is used to obtain the refractivity, pressure and temperature profiles (Kursinski et al., 1997). We refer to these as the standard RO thermodynamic profiles. The geometry found in the experiment is not a common RO configuration. Instead, the receiver is inside the atmosphere, i.e. on the ground, and therefore the tangent point-LEO trajectory is missing (see Fig. 2). The lack of symmetry and the non-existence of negative elevation observations mean that we cannot retrieve the standard thermodynamic profiles (Healy et al., 2002), which will be retrieved from the satellite in the future experiment.

Moreover, the fact that the receiver is on the ground means that the radio link crosses all the atmosphere layers during all of the observation time. In this configuration, the sounding of the atmosphere is different from an RO one. This has an important implication for the observables.

The polarimetric GNSS observable $\Delta \Phi$ is the difference between the carrier phase delay measured in the $H$ port and the one measured in the $V$ port. The observations in the $H$ and $V$ ports of the polarimetric antenna are independent, and therefore the receiver treats them separately. The GNSS receivers keep track of the total phase relative to their initial measurement, but the value associated with the first measurement is arbitrary (Blewitt, 1989). In this case, both signals $(H$ and $V$ ) suffer from this ambiguity (phase ambiguity, $b$ ) in their respective channel:

$$
\begin{aligned}
\Phi_{i}(t) & =\rho(t)+\rho_{\mathrm{atm}}(t)+\rho_{\mathrm{hyd}}^{i}(t)+\rho_{\mathrm{ion}}(t) \\
& +m^{i}(t)+d^{i}+C(t)+b^{i},
\end{aligned}
$$

where $\Phi$ is the measured carrier phase delay at the $i$ port ( $H$ or $V) . \rho$ is the geometry range between the satellite and the 


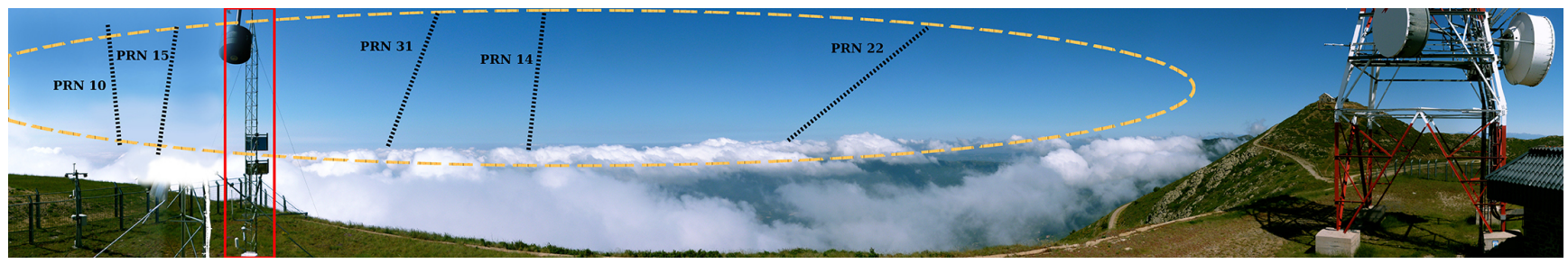

Figure 1. Panoramic view from the observation site. The field of view is the area comprising azimuths from $\sim 150^{\circ}$ (left) to $\sim 270^{\circ}$ (right), looking south. The yellow dashed line indicates the main lobe of the antenna (approximate). The black dashed lines represent the tracks of the GPS satellites followed: from left to right, PRN 10, 15, 31, 14 and 22. Multiple metallic elements seen in the field of view, such as the meteorological station (inside the red outline), the fence, the telecommunications antenna, and others not pictured (metallic shelter, antenna supports, etc.), could affect the GNSS signal in the form of multipath interference.

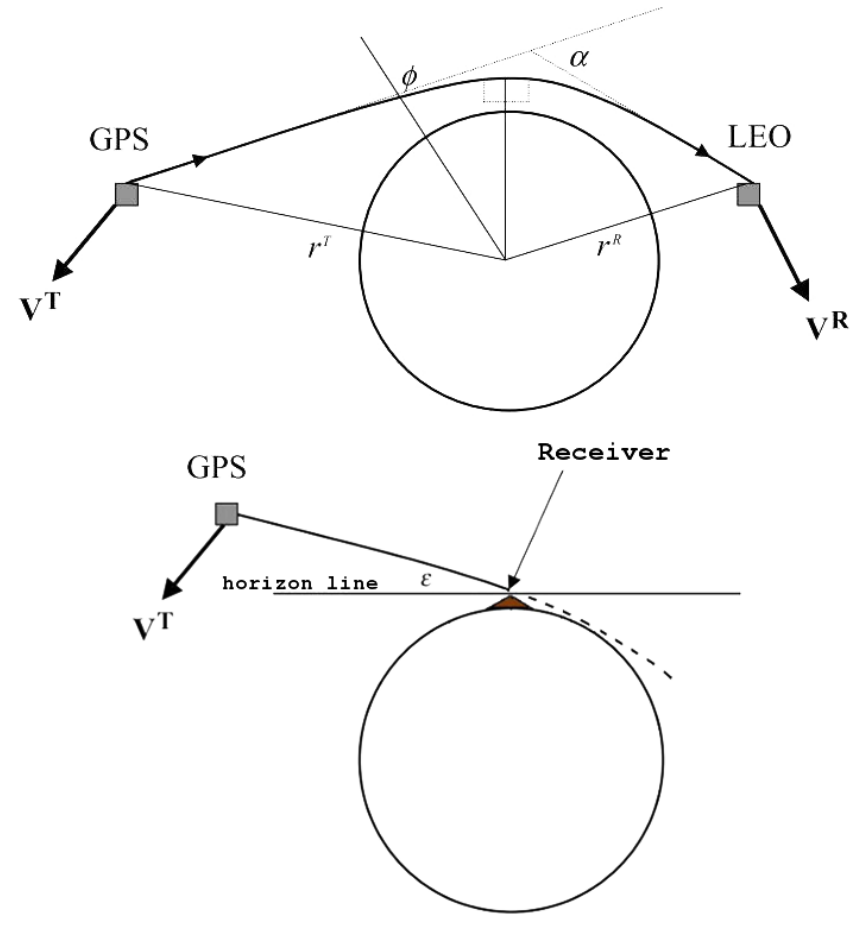

Figure 2. Top panel: standard radio occultation geometry. Bottom panel: GPS-receiver radio link in an on-ground receiver geometry, such as the one used in this experiment. $\epsilon$ indicates elevation. Figure adapted from Healy et al. (2002).

receiver since the initial measurement (the same for $H$ and $V), \rho_{\text {atm }}$ denotes the delay due to the neutral atmosphere that is equal in the $H$ and $V$ channels, $\rho_{\text {hyd }}$ is the phase delay due to the interaction with hydrometeors (the terms that we are interested in), and $\rho_{\text {ion }}$ denotes the ionospheric delay. $m$ represents the local multipath interference in each component, the term $d$ refers to the hardware effects of the receiver and the transmitter (such as noise, the effect of a possible difference in the cable's length, etc.), and $C$ represents the clock drifts and errors. $b$ is the arbitrary initial constant that does not depend on time. Most of these terms are common to both components; thus, the phase difference is
$\Delta \Phi(t)=\rho_{\mathrm{hyd}}^{H}(t)-\rho_{\mathrm{hyd}}^{V}(t)+m+b+d$,

where $m=m^{H}-m^{V}, b=b^{H}-b^{V}$ and $d=d^{H}-d^{V}$.

We do not have sufficiently precise pseudo-range measurements to solve the initial phase bias as is done in Blewitt (1989). The expected phase difference $\Delta \Phi$ is in the range of millimetres, while the pseudo-range accuracies are of the order of centimetres. This term $b$ changes in every arc of data (continuous tracking), and therefore the observation is not absolute but relative to the first measurement.

To avoid further problems, we identify the breaks in the tracking of the same PRN, and we separate them into continuous arcs. Every time that the track is lost, the receiver starts again with a new arbitrary constant. For each day, we only consider the longest arc and discard the rest. To enable comparison among different observations, we force each arc to have a 0 mean:

$\Delta \Phi^{\prime}(t)=\Delta \Phi(t)-\langle\Delta \Phi(t)\rangle$

This step homogenizes all the observations allowing the comparison among them. It removes the contribution from $b$ and $d$ terms, but it also erases any constant signature of the polarimetric measurement. Thus, any rain contribution in which depolarization is present from the beginning and remains until the end of the observation will be missed. In a satellite-to-satellite geometry (PAZ scenario), even without knowing the arbitrary initial constants we expect to be able to calibrate the initial phase, since at the beginning of the occultation, the radio link between the GPS and the LEO does not cross the atmosphere. A summary of the expected differences between the spaceborne mission and this ground experiment can be found in Table 1 .

\subsection{Local multipath interference}

Local multipath interference is the result of the combination of the signal from the satellite and one or more signals from the same source that have followed different paths to reach the receiver, for example, by being reflected on the ground or 
Table 1. Summary of the relevant differences expected between the ROHP-PAZ spaceborne experiment and the conducted ground-based field campaign.

\begin{tabular}{lll}
\hline Parameter & Ground-based experiment & ROHP-PAZ \\
\hline Initial phase delay & $\begin{array}{l}\text { unknown, need to subtract the mean value from } \\
\text { each measured arc (Eq. 4) }\end{array}$ & $\begin{array}{l}\text { calibrated from the polarimetric phase differ- } \\
\text { ence at highest layers of the atmosphere }\end{array}$ \\
\hline Local multipath interference & $\begin{array}{l}\text { multiple reflectors and environmental depen- } \\
\text { dency because of dry or wet changes in elec- } \\
\text { trical permittivity of soil and structures }\end{array}$ & $\begin{array}{l}\text { expected stable properties of local satellite } \\
\text { structure; no expected dependency on the envi- } \\
\text { ronment }\end{array}$ \\
\hline Thermodynamic profiles & $\begin{array}{l}\text { refractivity, pressure, temperature and humidity } \\
\text { cannot be extracted }\end{array}$ & $\begin{array}{l}\text { refractivity, pressure, temperature and humidity } \\
\text { can be derived }\end{array}$ \\
\hline
\end{tabular}

on a metallic structure. It affects the phase differently in the $H$ and in the $V$ components, giving a pattern that depends on the surrounding geometry, environmental conditions and position of the transmitter. The antenna is placed over a shelter, which has several metallic pieces. Moreover, there is a meteorological station a few metres from the experiment. Thus, the data suffer from a severe local multipath interference. If the reflecting process affected both $H$ and $V$ equally, this effect would cancel in $\Delta \Phi$. However, metallic structures with longitudinal edges might affect the scattering in the two polarizations differently.

The GPS satellites have an orbit period of one sidereal day. This implies that, in ideal conditions, the local multipath pattern ought to repeat after a sidereal day since the satellite is again in the same position with respect the observation site (it follows the same azimuth - elevation curve every sidereal day). To characterize and, to a large extent, remove the local multipath pattern from the signal, the time series of observations $\Delta \Phi_{\text {day }}^{\mathrm{PRN}}(t)$ are converted into elevation series $\Delta \Phi_{\text {day }}^{\mathrm{PRN}}(\epsilon)$. Time can be mapped onto elevation using the GPS orbit information that provides a precise GPS position for each time. This conversion allows the direct comparison among the observations from different days, making the signal only dependent on the satellite position.

Once the direct comparison is possible, the local multipath pattern can be found by performing the average and the standard deviation of the $\Delta \Phi_{\text {day }}^{\mathrm{PRN}}(\epsilon)$ for a given set of days. To account for all environmental conditions other than rain, the local multipath pattern is obtained using all the days identified as "no-rain" days. This identification is done taking into account information from two different sources: the ground weather station placed next to the observation site, and the radar reflectivity $\left(Z_{e}\right)$ from the weather radar of the area. If the ground weather station indicates that no rain was accumulated during the observation time and the weather radar indicates that no valid $Z_{e}$ values were present between the antenna and the GPS, the day is labelled as no-rain. More details about the meteorological information used in the data analysis can be found in Sect. 3 .
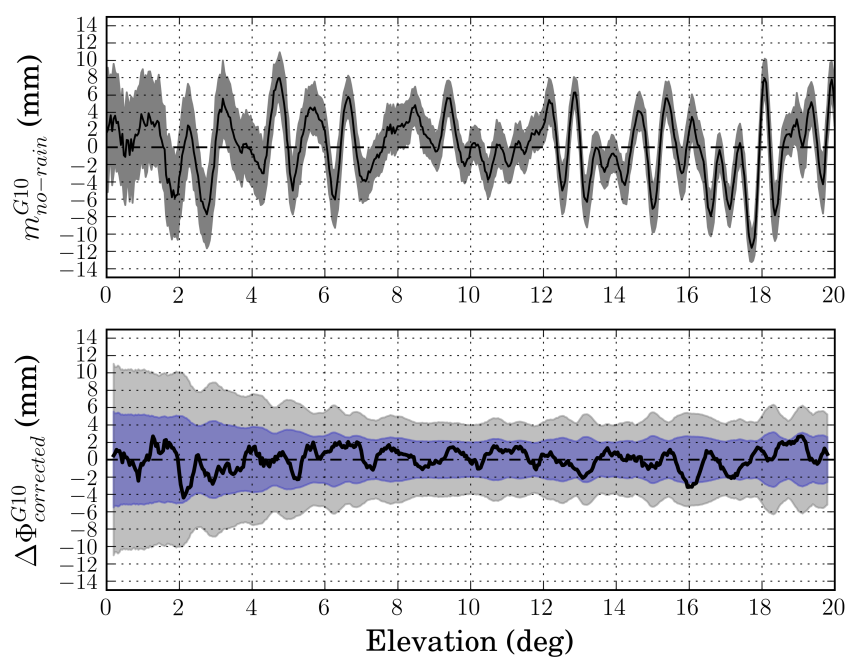

Figure 3. Examples of (top) local multipath pattern after applying Eq. (4) for PRN $10\left(m_{\text {no-rain }}^{\text {G10 }}, \sigma_{\text {no-rain }}^{\text {G10 }}\right)$, using a total of 132 days defined as no-rain days. Notice the large standard deviation at lower elevations and $\sigma_{\text {no-rain }}^{\mathrm{G} 10}$ of about $2 \mathrm{~mm}$ at higher elevations. Bottom panel: corrected $\Delta \Phi_{\text {day }}^{\mathrm{PRN}}(\epsilon)$ for 16 April 2014 (black line) after applying Eq. (5). The 1 and $2 \sigma$ thresholds (local multipath standard deviation) are represented in blue and grey, respectively.

The average $(m)$ and the standard deviation $(\sigma)$ of the norain days $\left(m_{\text {no-rain }}^{\text {PRN }}, \sigma_{\text {no-rain }}^{\mathrm{PRN}}\right.$ ) represent the local multipath pattern for no-rain days and can be seen in Fig. 3 (top). Note that the multipath pattern features vary between GPS transmitters because of different geometry and, therefore, different interaction with the nearby structures. Usually, $\sigma_{\text {no-rain }}^{\text {PRN }}$ is large at low elevations. This is due to a lower quality of the signal that has travelled a longer time through the atmosphere layers than of the rays at higher elevations. To obtain the final measurement, i.e. the one that will be analysed, this local multipath pattern is removed from the measured signal $\Delta \Phi^{\prime}(\epsilon)$ :

$\left.\Delta \Phi_{\text {day }}^{\mathrm{PRN}}(\epsilon)\right|_{\text {corrected }}=\left.\Delta \Phi_{\text {day }}^{\mathrm{PRN}}(\epsilon)\right|_{\text {observed }}-m_{\text {no-rain }}^{\mathrm{PRN}}(\epsilon)$. 

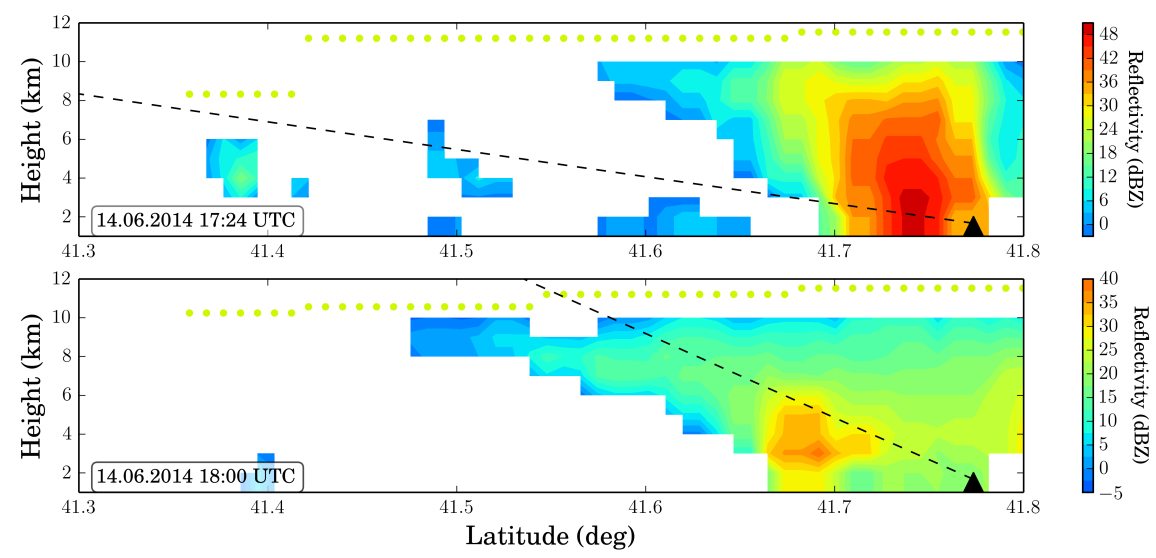

Figure 4. A vertical slice of radar reflectivity (shaded) at two epochs of a rising GNSS occultation event. The dashed black line is the projection of the ray trajectory as simulated with OAT ray tracer on the described plane, and the dots correspond to the cloud top phase (CP) products. In this case, all the green dots indicate ice at the top of the clouds.

The antenna pattern also affects the measurements differently in each component and induces a phase difference due to its different response to each polarization. Since the antenna is the PAZ's engineering model, its characteristics should be the same as those of the one mounted on the satellite, and its pattern is characterized in Cardellach et al. (2015). Its effect, though, is implicitly taken into account in the $m_{\text {no-rain }}^{\text {PRN }}$ term (it is constant in time and only depends on the satellite position), and therefore it is implicitly corrected by applying Eq. (5). Hereafter, the corrected measurement will be referred to as $\Delta \Phi_{\text {day }}^{\mathrm{PRN}}(\epsilon)$. An example of corrected $\Delta \Phi_{\text {day }}^{\mathrm{PRN}}$ is given in Fig. 3 (bottom).

\subsection{Ionosphere}

It is well known that the ionosphere affects the GPS signal carrier phase delays and pseudo-ranges. In terms of polarization, there are two effects relevant to the signals, the Faraday rotation and the Cotton-Mouton effect. The Faraday rotation is due to the longitudinal component of the Earth's magnetic field (longitudinal here meaning along the signal propagation direction), while the Cotton-Mouton effect is due to its transverse component (perpendicular to the propagation direction).

Faraday rotation changes the polarization axis of the propagating signals proportionally to the total electron content (TEC) crossed and the longitudinal component of the Earth's magnetic field. If the signal is transmitted at pure right-hand circular polarization (RHCP), then the rotation angle effect is the same in both components, $H$ and $V$, and therefore it should not be noticeable when taking the difference between the two signals.

On the other hand, the Cotton-Mouton effect could induce different phase delays in each component. According to Yeh et al. (1999), under the Earth's ionospheric conditions and frequency bands higher than $25 \mathrm{MHz}$, the Cotton-Mouton effect becomes significant only when the magnetic field is almost perpendicular to the propagation (see Figs. 4a and 5a for 25 and $1 \mathrm{MHz}$ in Yeh et al. (1999)). Otherwise, only the longitudinal component (Faraday rotation) is relevant. For example, despite the magnetic field being $\sim 80^{\circ}$ from the propagation, the Cotton-Mouton effect is still negligible.

Given the geometry of the GPS orbits, the experimental site location and antenna boresight orientation, these conditions (B at ionospheric altitudes perpendicular to GPS signal propagation) did not occur. Note that even for a LEO in polar orbit (such as the PAZ polarimetric-RO experiment will be), the probability of finding $\mathbf{B}$ perpendicular to the propagation direction is small.

\subsection{Measurement precision}

Even though it would be possible to determine the carrier phase measurement precision as in Cardellach et al. (2015), this would not be an actual value for the real precision of the polarimetric phase shift measurement in this experiment. Many factors, such as multipath interference, add dispersion to the observations and affect the actual precision of the measurement. These effects cannot be theoretically characterized and removed, but they have to be empirically determined.

Besides multipath interference, other effects are, for instance, a non-perfectly circular polarization of the emitted signals, which could lead to small polarimetric ionospheric effects (the waves emitted by the GPS satellites are RHCP within a small tolerance). Moreover, temperature variations in the surroundings could lead to small changes in the dielectric constant of the reflecting surfaces and therefore slightly modify the multipath pattern day after day. Among others, these effects add dispersion to the polarimetric phase shift measurement and cannot be disentangled from them. Therefore, they are ultimately included in the $\sigma_{\text {no-rain }}^{\text {PRN }}$ term in Eq. (5). 


\section{Meteorological weather data}

The objective of the analysis is to understand the new polarimetric observations, which requires collocated meteorological information. The weather radar of the area, in situ radiosonde data and Meteosat satellites measurements near the GNSS observational site are used in this study.

The Servei Meteorològic de Catalunya (METEOCAT) has a weather radar network covering the Catalan coastal area (Bech et al., 2004). We have access to the data from one of the radars, which has full coverage of the area under study. These radars are all Doppler systems, with one single polarization, operating at the $\mathrm{C}$ band $(5.6 \mathrm{GHz})$. The data provided consists of the radar reflectivity $\left(Z_{e}\right)$ in $\mathrm{dBZ}$ as a function of latitude, longitude and height. Its resolution is $1 \times 1 \times 1 \mathrm{~km}$ in a grid of $300 \times 300 \times 10 \mathrm{~km}(10 \mathrm{~km}$ referring to height $)$, and it was measured every $6 \mathrm{~min}$. Since it is not a polarimetric radar, we cannot extract information such as $K_{\mathrm{dp}}$ or $Z_{\mathrm{dr}}$, which would provide clues about the orientation of the particles. The minimum $Z_{e}$ value that is considered valid is $0 \mathrm{dBZ}$; below this the signal is considered noise and it is removed.

METEOCAT also has a network of ground stations that provide the accumulated precipitation, temperature and relative humidity in $30 \mathrm{~min}$ batches. Within a radius of $30 \mathrm{~km}$ around the observation site, there are five ground weather stations, with one located a few metres from the GNSS antennas. Through them we can have an approximation of the surface rain rate during the rain events.

Besides the radar and ground stations data, cloud type (CTY), cloud top phase (CP) and cloud top height (CTH) data products from the Satellite Application Facility on Support to Nowcasting and Very Short Range Forecasting (NWC-SAF) have been used. The data have been provided by the Agencia Estatal de Meteorología (AEMET) and the European Organisation for the Exploitation of Meteorological Satellites (EUMETSAT). These data products are a combination of satellite observations and NWP model simulations. The satellite observations are obtained by the Meteosat Second Generation (MSG) stationary meteorological satellites. They measure brightness temperatures and radiances with a radiometer at 12 different wavelengths (4 ranging from 0.4 to $1.6 \mu \mathrm{m}$ and 8 ranging from 3.9 to $13.4 \mu \mathrm{m})$. The horizontal resolution is $\sim 3 \mathrm{~km}$ and the products are available for the study area every $15 \mathrm{~min}$ (Aminou, 2002).

The collocated cloud observations from NWC-SAF (CTY, $\mathrm{CP}$ and $\mathrm{CTH}$ ) are then interpolated on to the GNSS ray trajectories. Unfortunately, these sets of data do not provide information about the orientation of the ice particles. Only those with their major axis oriented horizontally would induce a positive polarimetric signature. These data are mainly used to identify the top of the clouds and to identify ice above the maximum height of the radar products.

To complement all the information, we use the measurements provided by METEOCAT's radiosondes. These ra- diosondes are launched twice a day (00:00 and 12:00 UTC) at a distance of approximately $50 \mathrm{~km}$ to the south-east of the antenna and provide temperature, pressure and humidity as a function of height. With the limited two-time daily soundings, the temperature and refractivity profiles can be interpolated into the GNSS observation time.

Once all the information is recompiled, we can perform exact collocations of the GNSS polarimetric observations with the weather data. To do so, we first simulate the rays from the GPS to the antenna using a ray tracer called OAT, which solves the trajectory of each ray across the atmosphere characterized by the retrieved refractivity profiles (Aparicio and Rius, 2004). An illustration of the performed collocation can be seen in Fig. 4. Then, we interpolate all the weather information for each of the points of the ray trajectory. For this analysis, each ray consists of 500 points, each from the next by $\sim 0.52 \mathrm{~km}$. We simulate 501 rays, between 0 and $20^{\circ}$ of elevation.

\section{Statistical results: does rain induce polarimetric features?}

\subsection{Polarimetric signatures in $\Delta \Phi$ standard deviations}

Once the data have been preprocessed as described in Sect. 2, the analysis should determine whether the corrected $\Delta \Phi_{\text {day }}^{\mathrm{PRN}}(\epsilon)$ is affected by rain or not. To do so, corrected $\Delta \Phi_{\text {day }}^{\mathrm{PRN}}$ are grouped according to three different meteorological conditions. For each group, the standard deviation as a function of elevation $\sigma_{\text {met }}^{\mathrm{PRN}}(\epsilon)$ is computed. The three meteorological conditions and the corresponding $\sigma$ are as follows:

- dry days: days when the observation was made in a low relative humidity conditions (i.e. the relative humidity did not reach $100 \%$ ) according to the nearby ground weather station and without rain $\left(\sigma_{\mathrm{dry}}^{\mathrm{PRN}}(\epsilon)\right)$. No rain is stated when the nearby ground weather stations do not accumulate any rain during the observation time and the interpolation of the weather radar data along the GNSS rays does not cross any area where valid $Z_{e}$ values $\left(Z_{e}>0\right)$ are detected.

- wet days: days with high relative humidity (i.e. the relative humidity reaches $100 \%$ ) during or before the observation according to the nearby ground weather station, with rain before or after the observation, or with both $\left(\sigma_{\text {wet }}^{\mathrm{PRN}}(\epsilon)\right)$.

- rain days: days when the GNSS rays crossed an area where valid $Z_{e}$ values are detected by the weather radar $\left(\sigma_{\text {rain }}^{\mathrm{PRN}}(\epsilon)\right)$.

This classification has been done in order to compare different meteorological conditions. For example, high relative humidity conditions could have caused condensation, leading to a wet soil and different local multipath and antenna 
behaviour. The mean $\sigma$ across all elevation observations for each GNSS satellite during the three different meteorological conditions is summarized in Table 2.

It can be seen that dry days always present a lower $\sigma$ than the rest and that rain days exhibit the largest $\sigma$. The standard deviation for wet days is larger than for dry days, but the difference is less significant than for the rain days. There should not be any significant differences between wet and rain days, in terms of the immediate environment. For example, just after rain, the soil should be as wet as during the rain. Therefore, the larger $\sigma$ on rain days compared with the wet days indicates that factors other than the enhanced local multipath interference due to the wet soil on the rain days have contributed to the enhanced polarimetric signature.

To check whether this difference is enough to result in different populations (i.e. whether the cause of the different standard deviations is that we are under different scenarios and not that we are using a different sampling), we perform a statistical $F$ test (Walpole et al., 2012). We define the $f$ statistic as the ratio of the variances $\left(\sigma^{2}\right)$ of the samples that we are comparing and $P_{F}$ as the cumulative probability of $f$. We then compare the rain days with the no-rain days, where norain denotes all the wet and dry days. The results of $P_{F}$ are shown in Table 2. $P_{F}$ can be understood as the significance level that we are rejecting the null hypothesis, i.e. that the variances that we are comparing come from the same population. It can be seen that four out of the five analysed PRNs have a $P_{F}$ large enough to state that there is a difference in the standard deviation that could be related to rain.

Hereafter and for the rest of the analysis, the correction of the $\Delta \Phi_{\text {day }}^{\mathrm{PRN}}(\epsilon)$ is carried out as described in Eq. (5) using $m_{\text {no-rain }}^{\mathrm{PRN}}$, which is computed as in Sect. 2.2, taking all the dry and wet days defined in this section into account together.

\subsection{Phase difference as a function of elevation}

Examining each event individually, more features can be observed. To carry out such an analysis, we compare each observation $\Delta \Phi_{\text {day }}^{\mathrm{PRN}}(\epsilon)$ with the $\sigma_{\text {no-rain }}^{\mathrm{PRN}}(\epsilon)$. We define a $2 \sigma_{\text {no-rain }}^{\mathrm{PRN}}$ threshold to detect polarimetric signatures in the signal: statistically speaking, $\sim 95 \%$ of the data should be within $\pm 2 \sigma_{\text {no-rain }}^{\text {PRN }}$. Thus, the remaining $5 \%$ of the data points and those affected by some polarimetric feature should lie beyond $\pm 2 \sigma_{\text {no-rain }}^{\text {PRN }}$.

Lacking an absolute reference for the phase difference and in order to identify points overpassing the $\pm 2 \sigma_{\text {no-rain }}^{\text {PRN }}(\epsilon)$ threshold, we find the elevation point where the difference between $\Delta \Phi_{\text {day }}^{\mathrm{PRN}}(\epsilon)$ and $-2 \sigma_{\text {no-rain }}(\epsilon)$ is minimal, and we identify it as $\epsilon_{\min }$. Then, we subtract this difference from the observation and obtain the observation aligned in such a way that for each event its minimum lies on the line of the $-2 \sigma_{\text {no-rain }}$ threshold:

$\Delta \Phi_{S}(\epsilon)=\Delta \Phi(\epsilon)-\left(\Delta \Phi\left(\epsilon_{\min }\right)+2 \sigma_{\text {no-rain }}\left(\epsilon_{\min }\right)\right)$.
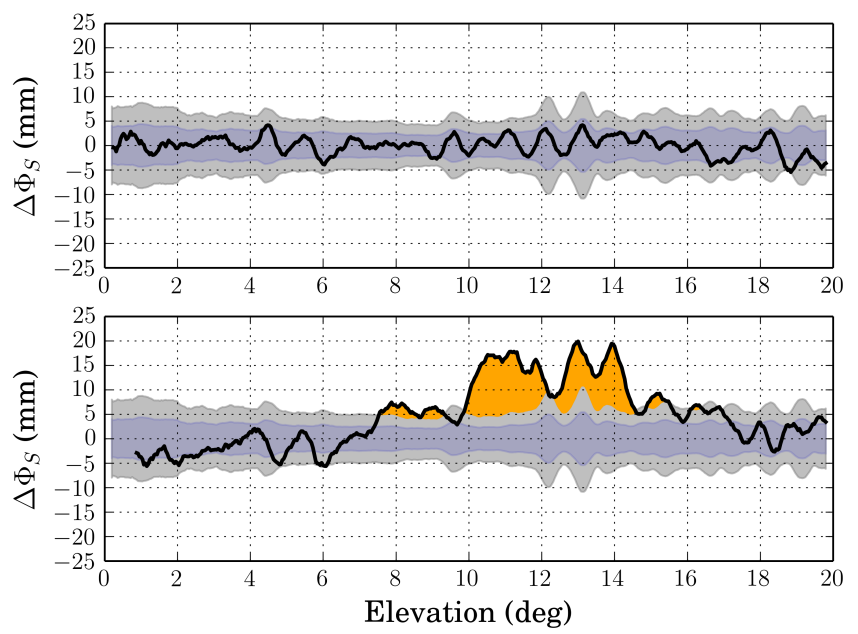

Figure 5. Examples of $\Delta \Phi_{S}(\epsilon)$ (black line), the $\pm \sigma_{\text {no-rain }}$ contour (blue) and the $\pm 2 \sigma_{\text {no-rain }}$ contour (grey), for two observations of the PRN G22 on 26 May 2014 (top) and 14 June 2014 (bottom). The top $\Delta \Phi_{S}(\epsilon)$ measurement is well inside the $2 \sigma$ contour, showing no polarimetric signatures. In the bottom panel, the case on 14 June 2014 shows large positive $\Delta \Phi_{S}(\epsilon)$. The value of $\Delta \Phi_{S}(\epsilon)$ above the $2 \sigma_{\text {no-rain }}$ threshold will hereafter be called $\Delta \Phi_{+}$and its area (orange zone) will be denoted by $A_{\Phi}$.

Defining $2 \sigma_{\text {no-rain }}^{\mathrm{PRN}}(\epsilon)$ as the no-rain noise level, $\Delta \Phi_{S}$ can be understood as a bias-corrected settled phase difference. After this correction, we can easily detect the points outside the $2 \sigma$ threshold. The region of $\Delta \Phi_{S}(\epsilon)$ above the $+2 \sigma_{\text {no-rain }}$ threshold is defined as follows:

$\Delta \Phi_{+}(\epsilon)=\left\{\begin{array}{lll}\Delta \Phi_{S}(\epsilon)-2 \sigma(\epsilon) & \text { if } \quad \Delta \Phi_{S}(\epsilon)>2 \sigma(\epsilon) \\ 0 & \text { if } \quad \Delta \Phi_{S}(\epsilon) \leq 2 \sigma(\epsilon) .\end{array}\right.$

$\Delta \Phi_{+}(\epsilon)$ would be the phase difference above the statistical no-rain noise level and its area is defined as $A_{\Phi}$ :

$A_{\Phi}=\int \Delta \Phi_{+}(\epsilon) d \epsilon$.

An example of $\Delta \Phi_{S}(\epsilon)$ and $A_{\Phi}$ is shown in the bottom plot in Fig. 5. In this procedure, we only consider the option of positive phase differences, as it is expected for rain effects (Cardellach et al., 2015). We have found 30 observations with $A_{\Phi}>0$, of which 28 correspond to rainy scenarios. This is the first direct observational evidence of the polarimetric signatures induced by precipitation conditions in the GNSS signals.

\section{Are the observed polarimetric features consistent with the models?}

In order to explain the observations, forward-scattering calculations have been performed. The aim is to simulate the effect of several kinds of hydrometeors, such as raindrops, 
Table 2. Summary of the standard deviation analysis for the polarimetric phase differences under three different meteorological conditions (dry, wet and rain days). $\overline{\sigma_{i}}$ and $N_{i}$ account for the mean standard deviation and the number of days used for each meteorological condition group $i . P_{F}$ is the cumulative probability associated with the $f$ statistic comparing the $\sigma$ of the rain and the no-rain (wet and dry) days. The $f$ statistic is the result of the $F$ test, and $P_{F}$ can be understood as the significance level at which we reject the null hypothesis that both samples come from the same population.

\begin{tabular}{ccccccc|c}
\hline PRN & $\overline{\sigma_{\text {dry }}}(\mathrm{mm})$ & $N_{\text {dry }}$ & $\overline{\sigma_{\text {wet }}}(\mathrm{mm})$ & $N_{\text {wet }}$ & $\overline{\sigma_{\text {rain }}}(\mathrm{mm})$ & $N_{\text {rain }}$ & $P_{F}$ \\
\hline G10 & 2.706 & 20 & 2.895 & 112 & 3.992 & 25 & 0.99 \\
G15 & 1.808 & 20 & 2.263 & 108 & 2.597 & 29 & 0.89 \\
G22 & 2.565 & 20 & 3.167 & 113 & 3.738 & 24 & 0.91 \\
G14 & 3.386 & 20 & 3.698 & 114 & 4.108 & 23 & 0.79 \\
G31 & 1.809 & 20 & 1.876 & 113 & 2.584 & 24 & 0.99 \\
\hline
\end{tabular}

pristine ice particles and melting ice particles, in order to cross-compare these with weather radar reflectivities, satellite observations and the phase differences measured.

First of all, the $K_{\mathrm{dp}}$ and the radar reflectivity factor $\left(Z_{e}\right)$ have been calculated for each hydrometeor type. These calculations have been done using the Discrete Dipole Scattering (DDScat) code (Draine and Flatau, 1994, 2013).

DDScat provides the phase lag efficiency factor $\left(Q_{\text {pha }}\right)$ for each polarimetric component $H$ and $V$. It is related to the forward-scattering amplitude $f_{\text {sca }}$ through $Q_{\text {pha }}=\frac{2 \pi}{k} \frac{\Re\left\{f_{\text {sca }}\right\}}{\pi a_{\text {eff }}^{2}}$. Thus, it can be used to calculate the $K_{\mathrm{dp}}$ :

$K_{\mathrm{dp}}=\frac{\lambda}{2 \pi} \int\left(Q_{\mathrm{pha}}^{H}-Q_{\mathrm{pha}}^{V}\right) \pi a_{\mathrm{eff}}^{2} N(D) \mathrm{d} D$,

where $a_{\text {eff }}$ is the equivolumetric radius of the particle, $N(D)$ is the particle size distribution, $D$ is the equivolumetric diameter and $K_{\mathrm{dp}}$ is given in millimetres per kilometre.

DDScat also provides the differential backscattering cross section normalized by $\pi a_{\text {eff }}^{2}$ :

$Q_{\mathrm{bk}}=\left.\frac{1}{\pi a_{\mathrm{eff}}^{2}} \frac{\partial \sigma_{\mathrm{sca}}}{\partial \Omega}\right|_{\Theta=180}$.

The backscattering cross section can then be obtained:

$\sigma_{\mathrm{bk}}=4 \pi Q_{\mathrm{bk}} \pi a_{\mathrm{eff}}^{2}$.

Using the $\sigma_{b k}$, the radar reflectivity factor $Z_{e}$ can be calculated as follows:

$Z_{e}=\frac{\lambda^{4}}{\pi^{5}\left|K_{\mathrm{w}}\right|^{2}} \int_{0}^{D_{\max }} \sigma_{b k}(D) N(D) \mathrm{d} D$,

where $\lambda$ is the wavelength and $K_{\mathrm{w}}=\left(m_{\mathrm{w}}^{2}-1\right) /\left(m_{\mathrm{w}}^{2}+2\right)$ and $m_{\mathrm{W}}$ is the complex refractive index of water (Smith, 1984).

$K_{\mathrm{dp}}$ is calculated for L-band frequency (GNSS observations) and $Z_{e}$ for C-band frequency (weather radar observations). This will allow us to relate the reflectivity from the weather radar in the $\mathrm{C}$ band with the GNSS observations in the $\mathrm{L}$ band.
The $N(D)$ that has been used is a gamma function of the form:

$N(D)=N_{0} D^{\mu} e^{-\Lambda D}$,

where $N_{0}$ is the scale parameter, $\Lambda$ is the slope parameter and $\mu$ is the shape parameter (Ulbrich, 1983). These are the three parameters of the gamma $N(D)$. The particle size distribution can be used to determine other quantities, such as the $K_{\text {dp }}$ (e.g. Eq. 9), $Z_{e}$ (e.g. Eq. 12), liquid or ice water content (LWC, IWC), effective particle diameter $\left(D_{\text {eff }}\right)$, mean weighted diameter $\left(D_{\mathrm{m}}\right)$, and rain rate $(R)$. Further details of the relation between these magnitudes and $N(D)$ can be found in the literature, for example in Williams et al. (2014).

Since there is no unique parameterization of $N(D)$ that applies to all scenarios, we generate a set of mathematically valid $\left(N_{0}, \Lambda, \mu\right)^{i}$ triplets, each one producing a different $N(D)^{i}$. Then, each triplet has an associated physical magnitude:

$\left(N_{0}, \Lambda, \mu\right)^{i} \rightarrow N(D)^{i} \rightarrow\left(K_{\mathrm{dp}}^{i}, Z_{e}^{i}, \mathrm{LWC}^{i}, D_{\mathrm{eff}}^{i}, D_{\mathrm{m}}^{i}, R^{i}, \ldots\right)$.

Depending on the hydrometeor being modelled, not all $N(D)$ parameters will be physically consistent, that is, fall in ranges that have been observed amongst various ground validation data (Williams et al., 2014). In the next section we describe the selection criteria for the valid ranges to choose among the possible $N(D)^{i}$.

\subsection{Modelled $A_{\Phi}:$ rain effects}

At the beginning of the campaign, only rain was expected to affect the polarimetric signal. To simulate the polarimetric rain effect, $Q_{\text {pha }}$ and $\sigma_{\text {bk }}$ have been calculated with DDScat using the predetermined oblate spheroid shapes, with $D$ ranging from 0.1 to $6 \mathrm{~mm}$ and the axis ratio (AR) following the Beard and Chuang (BC) relation (Beard and Chuang, 1987), as in Cardellach et al. (2015). The shapes are shown in Fig. 6 (left). Some constraints have been applied to the $\left(N_{0}, \Lambda, \mu\right)$ triplets in order to use only those producing physically valid quantities: we have limited $R$ to be as high as $70 \mathrm{~mm} \mathrm{~h}^{-1}$ as suggested by the meteorological ground sta- 


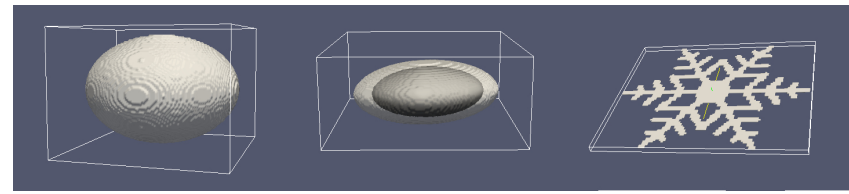

Figure 6. Particle shape models used in DDScat: oblate ellipsoid for raindrops (left); two concentric ellipsoids for melting ice particles, with an ice core and a water shell (middle); dendritic shape for pristine ice particles (right).

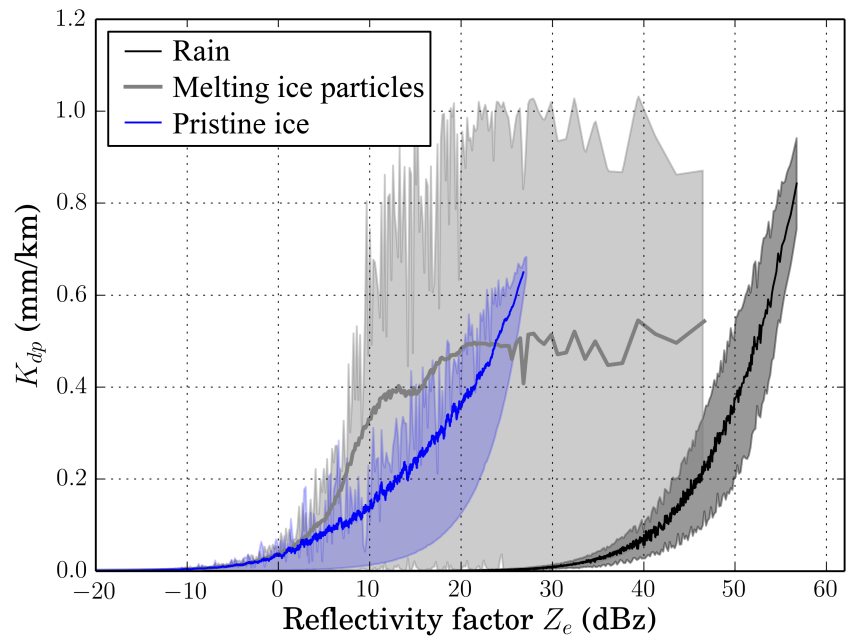

Figure 7. $K_{\mathrm{dp}}\left(Z_{e}\right)$ for all the possible physically valid $N(D)$ for each hydrometeor type: rain (black), melting ice particles (grey) and ice crystals (blue). Raindrops need high reflectivity to produce high $K_{\text {dp }}$, while ice crystals and melting ice particles can induce high values of $K_{\mathrm{dp}}$ at smaller values of $Z_{e}$. The thick lines overplotted represent the $Z_{e}-K_{\mathrm{dp}}$ relation used in this analysis for each hydrometeor type.

tions, and an upper limit of LWC is set to be $3 \mathrm{~g} \mathrm{~m}^{-3}$ according to the observational evidence of severe storms described in Black and Hallett (2012). All the parameter triplets producing quantities out of these ranges are discarded.

From the chosen $N(D)$ we derive $Z_{e}$ and $K_{\mathrm{dp}}$. All the valid $Z_{e}^{i}$ and $K_{\mathrm{dp}}^{i}$ for rain conditions are shown in black in Fig. 7. To relate the observations from the weather radar and the measurements from the polarimetric antenna, we need to use a $Z_{e}-K_{\mathrm{dp}}$ relation. It can be seen in Fig. 7 how a wide range of possible $K_{\mathrm{dp}}$ can be related to a given $Z_{e}$. For simplicity, we will use the $Z_{e}-K_{\mathrm{dp}}$ indicated by a thick line in Fig. 7.

We have simulated the expected $A_{\Phi}$ caused by rain for every GNSS measurements, using the radar $Z_{e}$ values interpolated to GNSS ray trajectories, and this $Z_{e}-K_{\mathrm{dp}}$ relation. The results are shown as black dots in Fig. 8. Despite the polarimetric signatures on rainy days, Fig. 8 shows that raindrops alone do not induce the large polarimetric signals observed (black dots in Fig. 8). Therefore, the effects of other hydrometeors must be taken into account.
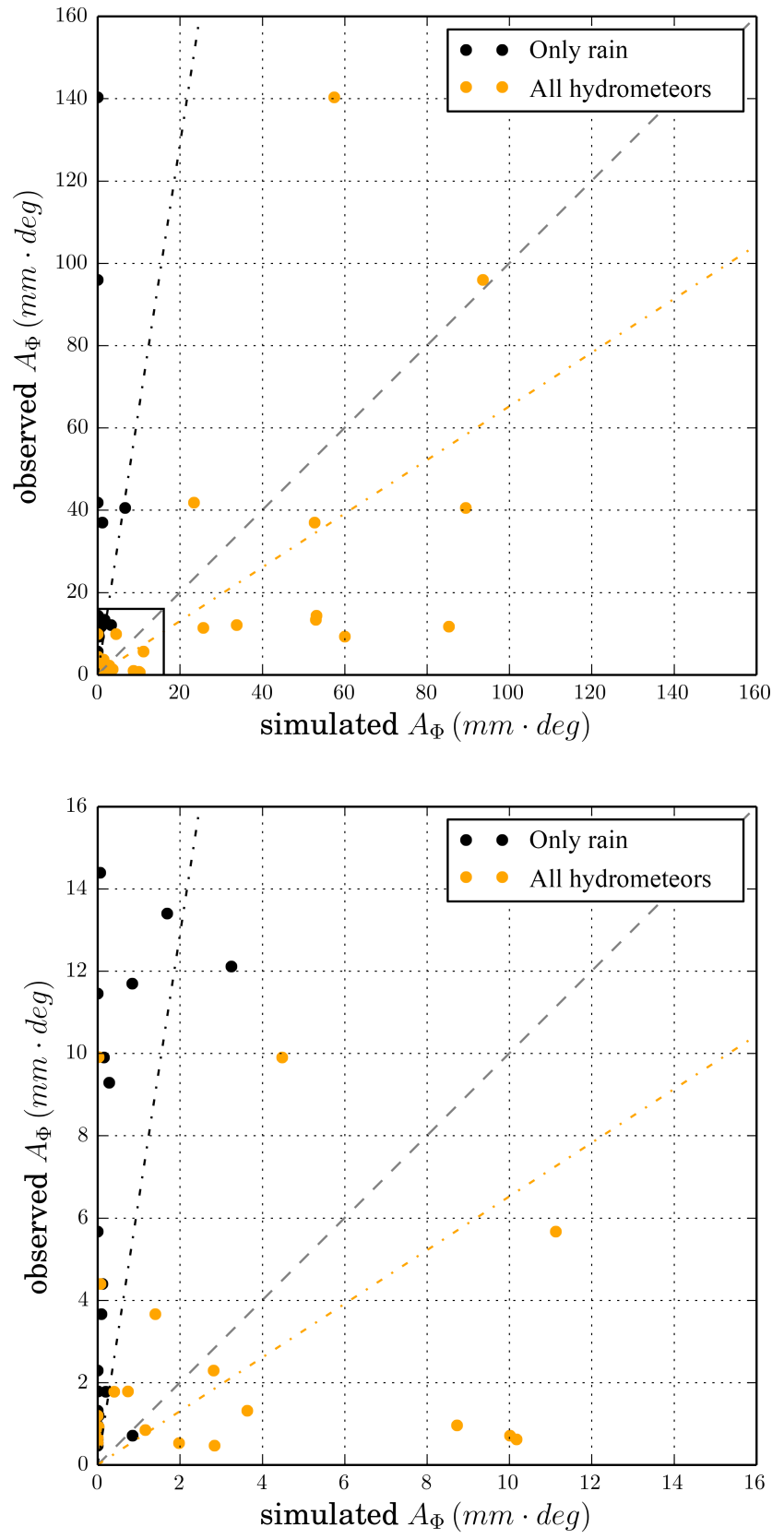

Figure 8. Top panel: observed versus simulated $A_{\Phi}$. Bottom panel: the area where $A_{\Phi}<16 \mathrm{~mm}$ deg in more detail. Black dots represent the simulated $A_{\Phi}$ using only raindrops, while orange dots represent the simulated $A_{\Phi}$ accounting for ice crystals and melting ice particles too. The dash-dot lines represent the best fitted line to the only rain $A_{\Phi}$ (black) and to the rain, ice and melting particles $A_{\Phi}$ (orange).

\subsection{Could ice and melting particles explain the large polarimetric signatures?}

We aim here to simulate the expected $A_{\Phi}$ induced by icy and melting particles. To simulate the ice particles, dendritic 
shapes have been used. Their characteristics are described in Liu (2008). For melting ice particles, two concentric ellipsoids have been used: the inner one made of pristine ice and the outer one of water. Both have the same axis ratio, ranging from 0.1 to 0.8 , and with $D$ ranging from 0.01 to $6 \mathrm{~mm}$. The water shell is considered to range between 5 and $10 \%$ of the volume of the inner core. Their shapes are sketched in Fig. 6 (centre and right).

A given ice-induced $Z_{e}$ can be explained by a range of ice particle characterizations, such as different combinations of canting angle, IWC, percentage of horizontally oriented particles with respect to randomly oriented ones, or the predominant sizes of the particles. This diversity of ice conditions relate to a diversity of $K_{\mathrm{dp}}$. This means that a given $Z_{e}$ links to many possible $K_{\mathrm{dp}}$ values. Since we want to keep this modelling simplistic to understand the polarimetric effect up to 1 order of magnitude and because we do not have ancillary information to properly characterize the ice properties, we have simulated this effect using only horizontally oriented dendrites, with a maximum IWC of $1 \mathrm{~g} \mathrm{~m}^{-3}$. Horizontal orientation is supported by many studies, for example Matrosov and Mace (2012) or Noel and Chepfer (2010). The IWC maximum is chosen according to the maximum values observed in Delanoë and Hogan (2010). The chosen $Z_{e}^{i}$ and $K_{\mathrm{dp}}^{i}$ for ice particles are shown in Fig. 7 in blue, and the $Z_{e}-K_{\mathrm{dp}}$ relation used for ice particles is highlighted with a thick blue line.

Melting ice particles have an even wider range of variability. As can be seen in Fig. 7 (in grey), the possible $Z_{e}^{i}$ and $K_{\mathrm{dp}}^{i}$ are widely spread. We have used the $Z_{e}-K_{\mathrm{dp}}$ relation indicated by a grey thick line when accounting for melting ice particles. As for rain and pristine ice, this relation is rather arbitrary, as we do not have the required ancillary ground-truth information to properly characterize these particles, and the goal is to explain, to 1 order of magnitude, the measurements.

We have separated the contribution of rain, ice, and melting ice particles according to the temperature. The temperatures are given by the METEOCAT's radiosondes, mentioned in Sect. 3. Noting that the radiosonde observation may differ in exact location and time, they are the closest to a true value of the temperature profiles. These radiosonde observations are in the GPS antenna field of view. For the cells above land (like the ones analysed here), METEOCAT profiles are less than $50 \mathrm{~km}$ away and temperatures above the boundary layer should be representative. The radar reflectivity measured at heights with temperatures above $1{ }^{\circ} \mathrm{C}$ is considered to come from rain. Particles between 1 and $-5^{\circ} \mathrm{C}$ are assumed to be melting ice particles. Below $-5^{\circ} \mathrm{C}$ they are assumed to be ice. Ice particles are assumed to be bigger between -5 and $-20^{\circ} \mathrm{C}$ because this region is considered to be the maximum dendritic growth zone (Kennedy and Rutledge, 2011). Above the radar measurements, ice contributions are assumed when the simulated ray intersects with ice regions, according to the combination of the cloud top phase and cloud top height products from the NWC-SAF. In this case, the particles are assumed to be smaller. We assume a thickness of the ice particle layer of about $2 \mathrm{~km}$, in agreement with Noel and Chepfer (2010).

In addition, the contribution to $A_{\Phi}$ due to ice and melting particles is only simulated when the observed $\Delta \Phi_{+}(\epsilon)$ is positive. The reason is that if there were no measurement of $\Delta \Phi_{+}(\epsilon)$, there would not be oriented crystals in the ray path, nor a contribution to $K_{\mathrm{dp}}$. The Cloud-Aerosol Lidar and Infrared Pathfinder Satellite Observation (CALIPSO) images show how only some regions of the clouds contain oriented ice crystals. This is consistent with discontinuous positive observations of $\Delta \Phi$, as made here. Unfortunately, no collocations were found between CALIPSO and the experiment.

The results for the simulated $A_{\Phi}$ taking into account the different hydrometeors are shown by orange dots in Fig. 8 . For every black dot (only rain simulated) an orange dot is included. Since these dots are intended to reproduce the same observed $A_{\Phi}$, there will be a black and an orange dot for every observed $A_{\Phi}$. A block diagram is shown in Fig. 9 to help the reader follow the steps that lead to the Fig. 8 results. All the data, information and relations used from the data acquisition to the final results are summarized in it.

Comparing the corresponding black and orange dots for a given observed $A_{\Phi}$, one may note how the simulated $A_{\Phi}$ increases significantly using all three hydrometeor types with respect to using only rain. Moreover, in most of the cases the simulated $A_{\Phi}$ is larger than the measured one (see the slope of the best fitted lines, dot-dashed in Fig. 8). This means that we tend to overestimate $A_{\Phi}$ in the simulations. Indeed, the particle characteristics that we have used in the simulations may increase the $K_{\mathrm{dp}}$ : the orientation of the particles is assumed to be horizontal (maximizing the polarimetric effect), and the type of particles is taken to be very asymmetric (when reality is more diverse).

Moreover, the model has been applied using the same $Z_{e}-K_{\mathrm{dp}}$ relation for each hydrometeor type, in every analysed rainy event. Fine tuning of the parameters for each individual observation would be needed in order to reproduce the observations fairly, but this would not be possible to validate due to the lack of ancillary independent information, and it is thus beyond the scope of this work. Nevertheless, it can be seen how the inclusion of icy and melting particles besides rain can explain the order of magnitude of the observations.

\subsection{Illustration cases}

In order to further check the internal consistency of the measurements, a comparison among several observations for different PRNs is performed during the evolution of heavy rain episodes. In this section we analyse three such episodes: events on 14 June, 22 August and 26 May 2014. To do so, we show the weather radar data, the observed phase difference above the noise level $\left(\Delta \Phi_{+}\right)$and the simulated $\Delta \Phi_{+}$. An example can be seen in Fig. 10. It corresponds to PRN 22 on 14 June 2014. 


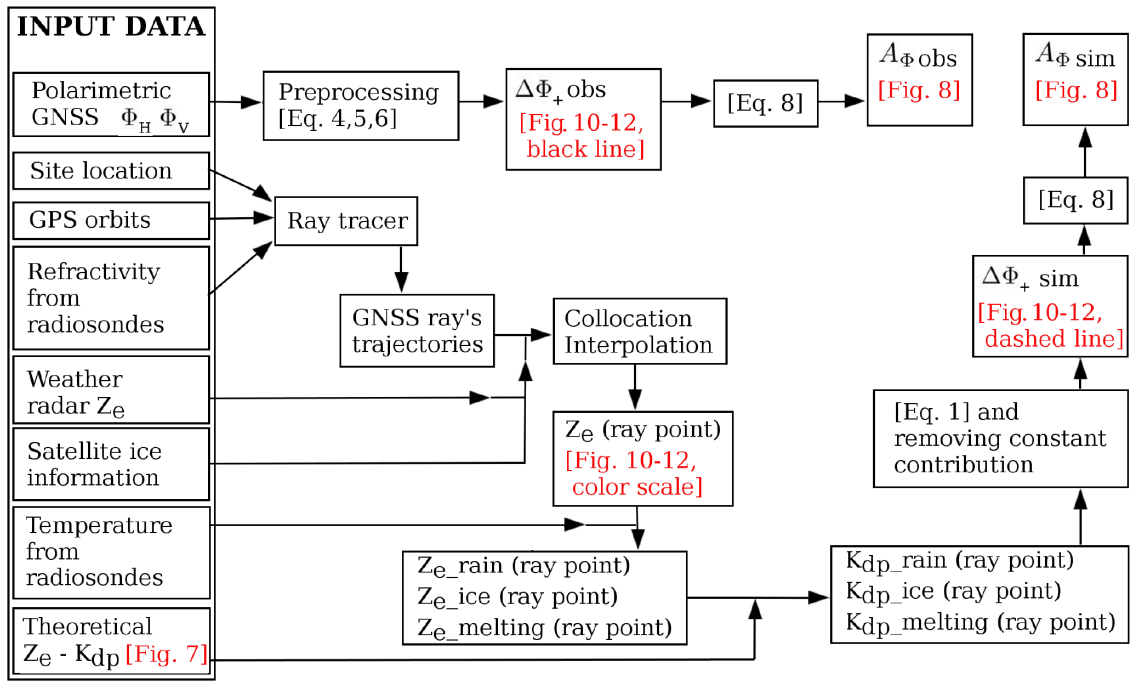

Figure 9. Block diagram showing all the data analysis and modelling process. All steps from the data acquisition to the final results are shown.

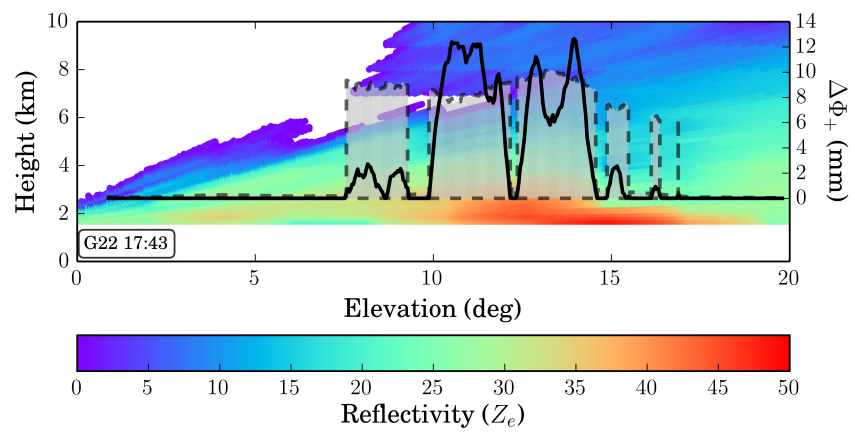

Figure 10. Each GNSS ray is identified by its elevation angle. Along a ray, each point can be identified by its height. The colour scale shows the weather radar reflectivity $Z_{e}$ interpolated along the GNSS rays. The black line is the observed $\Delta \Phi_{+}$(right $y$ axis). Simulation results performed as described in Sect. 5 are represented by dashed lines. In the regions where actual data showed $\Delta \Phi_{+}>0$, all hydrometeors are taken into account in the simulations. Otherwise, only rain is simulated. Note also that fully oriented ice crystals have been considered in the simulation; however, this orientation may not necessarily apply, as no information about the orientation of ice crystals is available.

The figure shows each GNSS ray identified by its elevation angle. Every point along the ray is associated with its height (left $y$ axis), and it is coloured according to the corresponding radar reflectivity $Z_{e}$ (from the interpolation between the GNSS rays and the weather radar). In addition, every elevation angle is associated with a $\Delta \Phi_{+}$measurement (an alongray integral measurement), and it is plotted as a thick black line with the values indicated by the right $y$ axis. The simulated $\Delta \Phi_{+}$is plotted with dashed lines along with the measured $\Delta \Phi_{+}$, and its values are also given by the right $y$ axis.
Therefore, in these figures the measured and simulated phase differences are overlaid on the radar reflectivity. A temporal series of such plots for heavy rain episodes is shown in Figs. 11 and 12.

Figure 11 corresponds to events on 14 June, 22 August and 26 May 2014 (same day represented in the same column):

- In the case of 14 June 2014, according to the nearby meteorological ground stations, there was a maximum accumulation of rain of $14 \mathrm{~mm}$ in $30 \mathrm{~min}$. This corresponds to peaks in rain rate higher than $28 \mathrm{~mm} \mathrm{~h}^{-1}$. Large positive $\Delta \Phi$ is present when large radar reflectivity $\left(Z_{e}\right)$ is accumulated at high altitudes. This is in agreement with the fact that rain alone produces lower polarimetric signatures than the ones detected with the present configuration.

- On 22 August 2014, the nearby meteorological ground stations suggest peaks in rain rate higher than $55 \mathrm{~mm} \mathrm{~h}^{-1}$ according to the accumulated precipitation over $30 \mathrm{~min}$. As in the previous case, positive $\Delta \Phi$ measurements are observed in the regions where significant $Z_{e}$ reaches high altitudes and where the temperature is around or below $0{ }^{\circ} \mathrm{C}$ (ice and melting particles).

- For the last case, on 26 May 2014, there was no such high rain rate peak, but significant $Z_{e}$ is also present at high altitudes, in agreement with the positive $\Delta \Phi$ observations.

Among all the studied cases (30), more than $93 \%$ (28) can be explained by the combined hydrometeor modelling, i.e. the modelling can reproduce the order of magnitude of the observations. An example of one of the two cases in which the simulations failed to explain the observations can be seen 

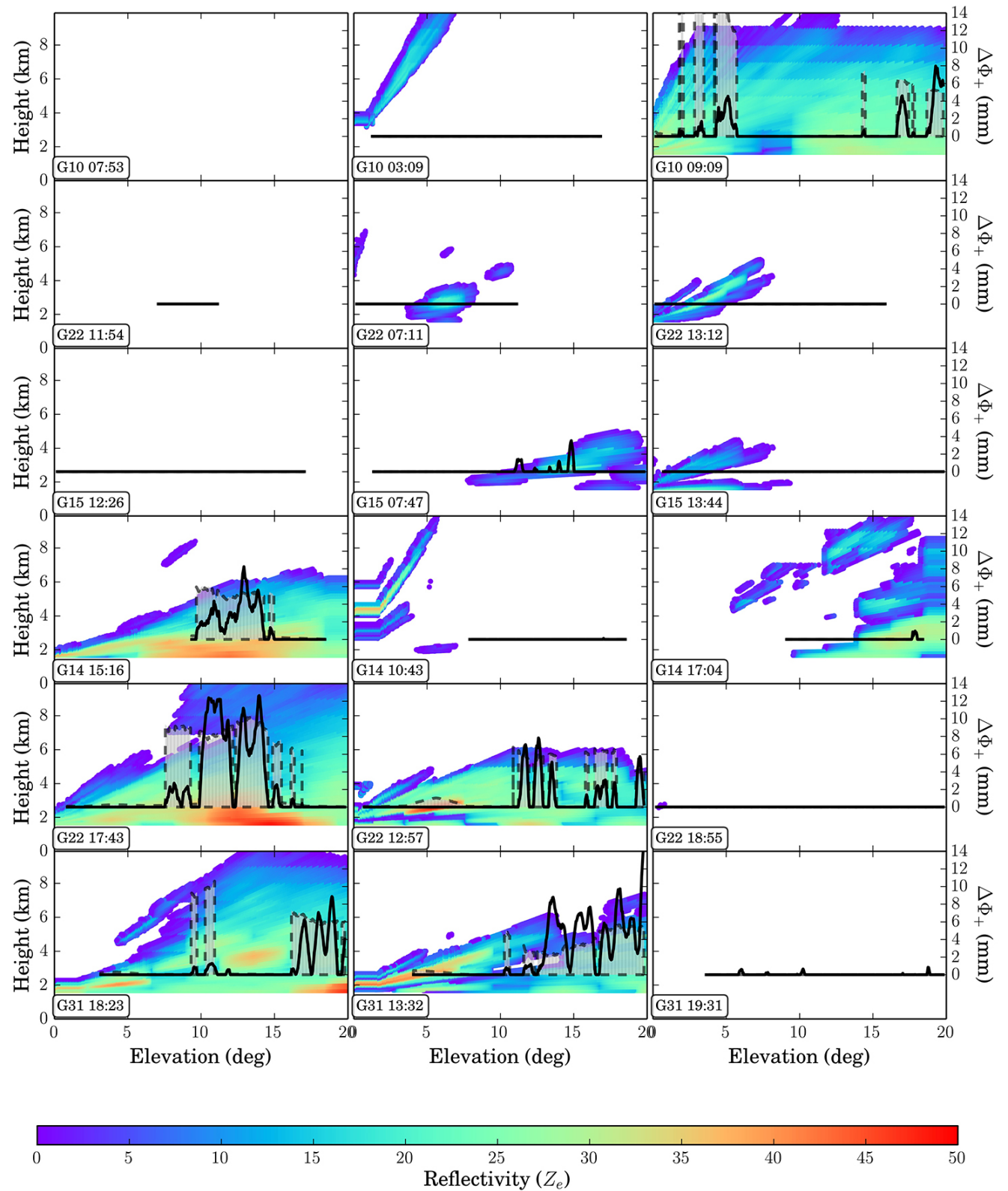

Figure 11. Rain episodes on 14 June 2014 (left), 22 August 2014 (middle) and 26 May 2014 (right). Each panel corresponds to a PRN, identified by the label in the lower left corner, along with the time when the satellite is at $10^{\circ}$ of elevation. Note that the radio link with different PRNs corresponds to different times and also different azimuth. The rain episodes are sorted according to time, with the earliest at the top. Content of each panel is explained in the caption to Fig. 10.

in Fig. 12: 9 July 2014. In this case, positive $\Delta \Phi$ measurements cannot be associated with any significant radar reflectivity nor with ice in the tops of the clouds crossed by the ray. Possible explanations could be some discrepancies due to missing observational data in the radar or errors in the temperature (which relies on the radiosonde interpolation) that might lead to a bad hydrometeor identification.

\section{Conclusions}

For the first time, GNSS occulting signals have been acquired using a dual-polarization antenna and the evidence of the polarimetric signature induced by hydrometeors in the GNSS signals has been presented. The technique, presented in Cardellach et al. (2015), will be tested from space aboard the PAZ low Earth orbiter. If successful, it will be possible to provide rain flags and potentially information about rain 

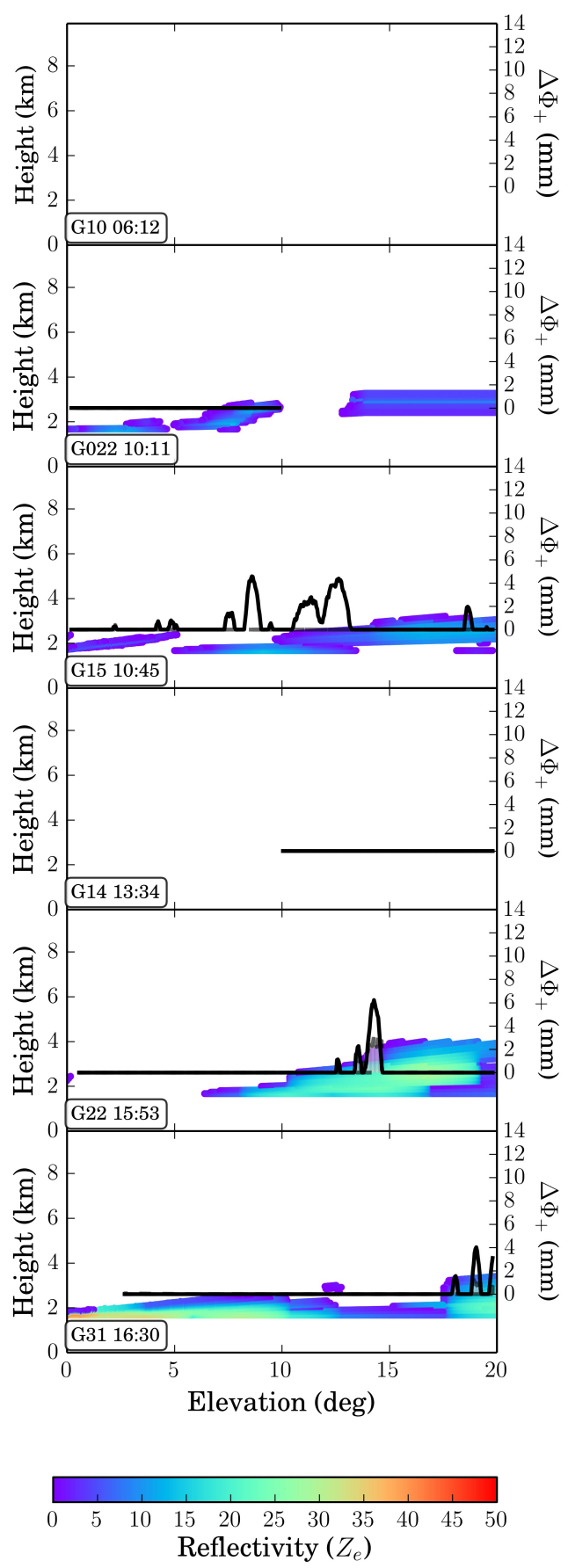

Figure 12. Same as Fig. 11 but for 9 July 2014. The signal in PRN G15 could not be explained by the model simulation.

structures, collocated with the standard RO thermodynamic profiles.

The experiment presented here was intended to characterize the phenomena that actually affect the polarimetric signatures. It has consisted of comparing the measurements of the polarimetric observable $\Delta \Phi$ under different weather condi- tions, trying to identify rain signatures. Data from five GNSS transmitters on $\sim 170$ different days have been analysed.

Many challenges have arisen in the data analysis process. Three main issues affect the data: the location of the antenna and receiver (low inside the atmosphere, no proper RO geometry), the phase ambiguity problem (linked to internal processing of the commercial receiver), and severe and varying local multipath interference (mostly due to the nearby environment, metallic towers and structures). These effects are not expected (or will be less severe) in the spaceborne mission. Regarding the location of the antenna and receiver, the future experiment will be in space and therefore outside the atmosphere. Being outside the atmosphere will allow a better calibration of the signals thanks to the scanning geometry: a vertical descent from the outer layers approaching the Earth's surface. Thus, at the beginning of the observation, there are no depolarizing effects, and it will be possible to define the initial state (calibration of the polarimetric phase measurement). Phase ambiguity will be solved, and absolute measurements will be possible, unlike in this experiment. Moreover, with the satellite, we expect the local multipath interference to be smaller, and most importantly, it will not change with the environmental conditions.

After analysing the data, two main conclusions can be extracted. From a general point of view, rain scenarios affect the polarimetric observables. The standard deviation of the mean $\Delta \Phi$ for dry, wet and rain days have been examined. For environmental reasons (wet soil, increase in the reflectivity, etc.), the $\sigma$ for wet and rain days is higher than for dry days. However, the increase in the $\sigma_{\text {rain }}$ with respect to $\sigma_{\text {dry }}$ is between 20 and the $40 \%$ larger than the increase in $\sigma_{\text {wet }}$ with respect to $\sigma_{\text {dry }}$.

This could empirically answer one of the questions for which we were seeking an answer: are radio links crossing rain cells affected by any depolarization affect? And if so, is it detectable? According to the $\sigma$ behaviour under the different weather and environmental conditions, we can state that under rain scenarios, the measured $\Delta \Phi$ suffers from higher variability and the difference to other scenarios is noticeable.

A more detailed analysis of the $\Delta \Phi(\epsilon)$ has been performed for each individual observation. $\Delta \Phi$ above the defined $2 \sigma$ threshold and the computed $A_{\Phi}$ have been compared with simulated results. Simulated $K_{\mathrm{dp}}$ using the collocated radar reflectivity has shown that raindrops induce an effect much lower than the measurements. This indicates that other phenomena are inducing polarimetric signatures too. This is an important point in view of the future analysis of the spaceborne ROHP-PAZ data.

Ice crystals and melting ice particles have been added to the modelling, using temperature information and satellite imagery to distinguish between hydrometeors. Simulations of these particles have been kept very simplistic due to the number of possible parameters involved in the modelling and the lack of information to validate them. The goal at this stage is to identify the sources of polarimetric signatures. 
The simulations have shown that, in most of the cases, the measured $\Delta \Phi$ could be explained by the $K_{\mathrm{dp}}$ induced by all possible hydrometeors.

Simulated $\Delta \Phi$ and $A_{\Phi}$ with all the hydrometeors are usually above the measured values. Fine tuning of the parameters involved in the modelling would be needed in order to match the observations, but the parameters are impossible to validate in this particular experiment. Microphysical analysis of the precipitation and inversion procedure development are left for later studies with RO data more suitable than mountain-top occultations. Nevertheless, the results obtained here represent the first empirical evidence that hydrometeors induce measurable polarimetric signatures in occulting GNSS signals according to the theoretical analysis in Cardellach et al. (2015). These results help us to understand the types of processes affecting the data from the future polarimetric RO experiment aboard PAZ. They additionally show the potential capability of polarimetric RO to sense complex precipitable structures, information that will be provided along with thermodynamic profiles and increase the applications of the RO technique.

Acknowledgements. This study was conducted under the Spanish ACI2010-1089 and AYA2011-29183-C02-02 grant, with contributions from EUMETSAT's ROM SAF CDOP2. R. Padullés is supported by the Spanish FPI program and he also received three JPL Vistor Student Researcher Program invitations. E. Cardellach is supported by the Spanish Ramon y Cajal programme. Work contributed by C. O. Ao, M. de la Torre Juárez, and F. J. Turk was performed at the Jet Propulsion Laboratory, California Institute of Technology, under a contract with the National Aeronautics and Space Administration. Funding support from NASA ROSES Grants NNH14ZDA001N-ESUSPI, NNH10ZDA001NGEOIM, and NNH11ZDA001N-ESI is gratefully acknowledged. A relevant contribution to the logistics and implementation of the experimental site was made by Adriano Camps' group at the Remote Sensing Lab, department of Teoria del Senyal i Comunicacions, Universitat Politècnica de Catalunya, under grant AYA2011-29183C02-01. Some of these grants are partially funded by the European ERDF/FEDER programme.

The Helmholtz Centre German Research Centre for Geosciences (GFZ Potsdam) provided the Javad GPS receiver. Results were obtained thanks to the meteorological data provided by the Servei Meteorològic de Catalunya (METEOCAT), the Agencia Estatal de Meteorología, Ministerio de Agricultura, Alimentación y Medio Ambiente (AEMET) and the European Organisation for the Exploitation of Meteorological Satellites (EUMETSAT). The experimental site belongs to the Spanish Ministry of Defence, Estado Mayor de la Defensa (EMAD).

The authors want to thank the two anonymous reviewers and the editor whose comments improved the manuscript. Bruce T. Draine and Piotr J. Flatau are gratefully acknowledged for providing the DDScat code. Particle shapes for DDScat code were provided by G. Liu and Ryan Honeyager from Florida State University. The authors want to thank G. Hajj for interesting discussions about the ionosphere.
Edited by: G. Vaughan

\section{References}

Aminou, D. M.: MSG's SEVIRI Instrument, ESA Bulletin, 111, 15-17, 2002.

Aparicio, J. M. and Rius, A.: A raytracing inversion procedure for the extraction of the atmospheric refractivity from GNSS travel-time data, Phys. Chem. Earth, Pt A/B/C, 29, 213-224, doi:10.1016/j.pce.2004.01.008, 2004.

Baldini, L. and Gorgucci, E.: Identification of the melting layer through dual-polarization radar measurements at vertical incidence, J. Atmos. Ocean. Technol., 23, 829-839, doi:10.1175/JTECH1884.1, 2006.

Beard, K. V. and Chuang, C.: A new model for the equilibrium shape of raindrops, J. Atmos. Sci., 44, 1509-1524, doi:10.1175/1520-0469(1987)044<1509:ANMFTE>2.0.CO;2, 1987.

Bech, J., Vilaclara, E., Pineda, N., Rigo, T., Lopez, J., O’Hora, F., Lorente, J., Sempere, D., and Fabregas, F. X.: The weather radar network of the Catalan Meteorological Service: description and applications, in: Proceedings of ERAD, vol. 2, pp. 416-420, 2004.

Black, R. A. and Hallett, J.: Rain Rate and Water Content in Hurricanes Compared with Summer Rain in Miami, Florida, J. Appl. Meteorol. Clim., 51, 2218-2235, doi:10.1175/JAMC-D11-0144.1, 2012.

Blewitt, G.: Carrier phase ambiguity resolution for the Global Positioning System applied to geodetic baselines up to $2000 \mathrm{~km}$, J. Geophys. Res., 94, 10187-10203, doi:10.1029/JB094iB08p10187, 1989.

Bringi, V. N. and Chandrasekar, V.: Polarimetric weather radar; principles and applications, Cambridge University Press, 2001.

Cardellach, E., Tomás, S., Oliveras, S., Padullés, R., Rius, A., de la Torre-Juárez, M., Turk, F. J., Ao, C. O., Kursinski, E. R., Schreiner, W., Ector, D., and Cucurull, L.: Sensitivity of PAZ LEO Polarimetric GNSS Radio-Occultation Experiment to Precipitation Events, IEEE Trans. Geosci. Remote Sens., 53, 190206, doi:10.1109/TGRS.2014.2320309, 2015.

Cucurull, L. and Derber, J. C.: Operational Implementation of COSMIC Observations into NCEP's Global Data Assimilation System, Weather Forecast., 23, 702-711, doi:10.1175/2008WAF2007070.1, 2008.

Delanoë, J. and Hogan, R. J.: Combined CloudSat-CALIPSOMODIS retrievals of the properties of ice clouds, J. Geophys. Res., 115, D00H29, doi:10.1029/2009JD012346, 2010.

Draine, B. T. and Flatau, P. J.: Discrete-dipole approximation for scattering calculations, J. Opt. Soc. Am. A, 11, 1491, doi:10.1364/JOSAA.11.001491, 1994.

Draine, B. T. and Flatau, P. J.: User Guide for the Discrete Dipole Approximation Code DDSCAT 7.3, arXiv preprint, 3, http:// arxiv.org/abs/1305.6497, 2013.

Ducrocq, V., Braud, I., Davolio, S., Ferretti, R., Flamant, C., Jansa, A., Kalthoff, N., Richard, E., Taupier-Letage, I., Ayral, P. A., Belamari, S., Berne, A., Borga, M., Boudevillain, B., Bock, O., Boichard, J. L., Bouin, M. N., Bousquet, O., Bouvier, C., Chiggiato, J., Cimini, D., Corsmeier, U., Coppola, L., Cocquerez, 
P., Defer, E., Delanoë, J., Di Girolamo, P., Doerenbecher, A., Drobinski, P., Dufournet, Y., Fourrié, N., Gourley, J. J., Labatut, L., Lambert, D., Le Coz, J., Marzano, F. S., Molinié, G., Montani, A., Nord, G., Nuret, M., Ramage, K., Rison, B., Roussot, O., Said, F., Schwarzenboeck, A., Testor, P., Van Baelen, J., Vincendon, B., Aran, M., and Tamayo, J.: HyMeX-SOP1, the field campaign dedicated to heavy precipitation and flash flooding in the northwestern Mediterranean, B. Am. Meteorol. Soc., 95, 10831100, doi:10.1175/BAMS-D-12-00244.1, 2014.

Healy, S. B., Haase, J., and Lesne, O.: Letter to the Editor Abel transform inversion of radio occultation measurements made with a receiver inside the Earth's atmosphere, Ann. Geophys., 20, 1253-1256, doi:10.5194/angeo-20-1253-2002, 2002.

Healy, S. B., Jupp, A. M., and Marquardt, C.: Forecast impact experiment with GPS radio occultation measurements, Geophys. Res. Lett., 32, L03804, doi:10.1029/2004GL020806, 2005.

Kennedy, P. C. and Rutledge, S. A.: S-Band Dual-Polarization Radar Observations of Winter Storms, J. Appl. Meteorol. Clim., 50, 844-858, doi:10.1175/2010JAMC2558.1, 2011.

Kursinski, E. R., Hajj, G. A., Schofield, J. T., Linfield, R. P., and Hardy, K. R.: Observing Earth's atmosphere with radio occultation measurements using the Global Positioning System, J. Geophys. Res., 102, 23429-23465, doi:10.1029/97JD01569, 1997.

Liu, G.: A Database of Microwave Single-Scattering Properties for Nonspherical Ice Particles, B. Am. Meteorol. Soc., 89, 15631570, doi:10.1175/2008BAMS2486.1, 2008.

Matrosov, S. Y.: Radar reflectivity in snowfall, IEEE Trans. Geosci. Remote Sens., 30, 454-461, doi:10.1109/36.142923, 1992.

Matrosov, S. Y. and Mace, G. G.: Observations of ice crystal habits with a scanning polarimetric W-band radar at slant linear depolarization ratio mode, J. Atmos. Ocean. Technol., 29, 989-1008, doi:10.1175/JTECH-D-11-00131.1, 2012.

Noel, V. and Chepfer, H.: A global view of horizontally oriented crystals in ice clouds from Cloud-Aerosol Lidar and Infrared Pathfinder Satellite Observation (CALIPSO), J. Geophys. Res., 115, doi:10.1029/2009JD012365, 2010.

Rocken, C., Anthes, R. A., Exner, M., Hunt, D., Sokolovskiy, R., Ware, R. H., Gorbunov, M., Schreiner, W., Feng, D., Herman, B. M., Kuo, Y. H., and Zou, X.: Analysis and validation of GPS/MET data in the neutral atmosphere, J. Geophys. Res., 102, 29849-29866, doi:10.1029/97JD02400, 1997.
Ryzhkov, A. V. and Zrnić, D. S.: Polarimetric method for ice water content determination, J. Appl. Meteorol., 37, 125-134, 1998.

Smith, P. L.: Equivalent Radar Reflectivity Factors for Snow and Ice Particles, J. Clim. Appl. Meteorol., 23, 1258-1260, doi:10.1175/1520-0450(1984)023<1258:ERRFFS>2.0.CO;2, 1984.

Steiner, A. K., Lackner, B. C., Ladstdter, F., Scherllin-Pirscher, B., Foelsche, U., and Kirchengast, G.: GPS radio occultation for climate monitoring and change detection, Radio Science, 46, 1-17, doi:10.1029/2010RS004614, 2011.

Trömel, S., Kumjian, M. R., Ryzhkov, A. V., Simmer, C., and Diederich, M.: Backscatter differential phase-estimation and variability, J. Appl. Meteorol. Clim., 52, 2529-2548, doi:10.1175/JAMC-D-13-0124.1, 2013.

Ulbrich, C. W.: Natural Variations in the Analytical Form of the Raindrop Size Distribution, J. Clim. Appl. Meteorol., 22, 1764-1775, doi:10.1175/15200450(1983)022<1764:NVITAF>2.0.CO;2, 1983.

Vivekanandan, J., Bringi, V. N., Hagen, M., and Meischner, P.: Polarimetric radar studies of atmospheric ice particles, IEEE Trans. Geosci. Remote Sens. 32, 1-10, doi:10.1109/36.285183, 1994.

Walpole, R. E., Myers, R. H., Myers, S. L., and Ye, K.: Probability and Statistics for Engineers and Scientists, Prentice Hall, Boston, 9th Edn., 2012.

Williams, C. R., Bringi, V. N., Carey, L. D., Chandrasekar, V., Gatlin, P. N., Haddad, Z. S., Meneghini, R., Joseph M., S., Nesbitt, S. W., Petersen, W. A., Tanelli, S., Tokay, A., Wilson, A., and Wolff, D. B.: Describing the Shape of Raindrop Size Distributions Using Uncorrelated Raindrop Mass Spectrum Parameters, J. Appl. Meteorol. Climatol., 53, 1282-1296, doi:10.1175/JAMC-D-13-076.1, 2014.

Yeh, K. C., Chao, H. Y., and Lin, K. H.: A study of the generalized Faraday effect in several media, Radio Science, 34, 139153, doi:10.1029/98RS02442, 1999. 